\title{
Functional phenotypes determined by fluctuation- based clustering of lung function measurements in healthy and asthmatic cohort participants
}

\author{
Edgar Delgado-Eckert, ${ }^{1,2}$ Oliver Fuchs, ${ }^{3,4}$ Nitin Kumar, ${ }^{1}$ Juha Pekkanen, 5,6 \\ Jean-Charles Dalphin, ${ }^{7}$ Josef Riedler, ${ }^{8}$ Roger Lauener, ${ }_{1}^{9}$ Michael Kabesch, ${ }^{10,11}$ \\ Maciej Kupczyk, ${ }^{12,13}$ Sven-Erik Dahlen, ${ }^{12}$ Erika von Mutius, ${ }^{3}$ Urs Frey ${ }_{1}{ }^{1}$ the PASTURE \\ and BIOAIR Study groups
}

\begin{abstract}
- Additional material is published online only. To view please visit the journal online (http://dx.doi.org/10.1136/ thoraxjn-2016-209919).
\end{abstract}

For numbered affiliations see end of article.

Correspondence to Dr Edgar Delgado-Eckert, University of Basel, University Children's Hospital (UKBB), Spitalstrasse 33, Postfach, Basel 4031, Switzerland; edgar. delgado-eckert@unibas.ch

OF and NK contributed equally.

Some of the results of these studies have been previously reported in the form of an abstract (see reference 32).

Received 23 December 2016 Revised 11 July 2017 Accepted 31 July 2017 Published Online First 1 December 2017

CrossMark

To cite: Delgado-Eckert $\mathrm{E}$, Fuchs 0 , Kumar $\mathrm{N}$, et al.

Thorax 2018;73:107-115.

\begin{abstract}
Rationale Asthma is characterised by inflammation and reversible airway obstruction. However, these features are not always closely related. Fluctuations of daily lung function contain information on asthma phenotypes, exacerbation risk and response to long-acting $\beta$-agonists. Objectives In search of subgroups of asthmatic participants with specific lung functional features, we developed and validated a novel clustering approach to asthma phenotyping, which exploits the information contained within the fluctuating behaviour of twice-daily lung function measurements.

Methods Forced expiratory volume during the first second $\left(\mathrm{FEV}_{1}\right)$ and peak expiratory flow (PEF) were prospectively measured over 4 weeks in 696 healthy and asthmatic school children (Protection Against Allergy - Study in Rural Environments (PASTURE)/EFRAIM cohort), and over 1 year in 138 asthmatic adults with mild-to-moderate or severe asthma (Pan-European Longitudinal Assessment of Clinical Course and BIOmarkers in Severe Chronic AIRway Disease (BIOAIR) cohort). Using enrichment analysis, we explored whether the method identifies clinically meaningful, distinct clusters of participants with different lung functional fluctuation patterns.
\end{abstract}

Measurements and main results In the PASTURE/ EFRAIM dataset, we found four distinct clusters. Two clusters were enriched in children with wellknown clinical characteristics of asthma. In cluster 3, children from a farming environment predominated, whereas cluster 4 mainly consisted of healthy controls. About $79 \%$ of cluster 3 carried the asthma-risk allele rs7216389 of the 17q21 locus. In the BIOAIR dataset, we found two distinct clusters clearly discriminating between individuals with mild-to-moderate and severe asthma.

Conclusions Our method identified dynamic functional asthma and healthy phenotypes, partly independent of atopy and inflammation but related to genetic markers on the 17q21 locus. The method can be used for disease phenotyping and possibly endotyping. It may identify participants with specific functional abnormalities, potentially needing a different therapeutic approach.

\section{INTRODUCTION}

Approaches to identifying phenotypes or endotypes in asthma have become increasingly relevant. $^{1-7}$ Cluster analysis using clinical, atopic, and

\section{Key messages}

What is the key question?

- Using a novel approach to asthma phenotyping based on clustering of daily lung function fluctuations, we aim to determine whether we can identify asthma patients with specific functional characteristics in response to the time-varying stimuli of the environment to which they are exposed.

What is the bottom line?

- Lung function fluctuations reveal asthma phenotypes that are partly independent of the inflammatory disease process but strongly related to airway mechanics and bronchodilator response.

Why read on?

- This novel, fluctuation-based clustering technique may help identify asthmatics with functional abnormalities, potentially benefitting from alternative therapeutic schemata, rather than anti-inflammatory treatment only.

inflammatory biomarkers has facilitated phenotyping in selected cross-sectional asthma studies. ${ }^{8-12}$ However, in the majority of published approaches, the characterising parameters are only assessed at a single point in time,${ }^{8-18}$ yielding phenotypes that might not remain stable as time progresses. ${ }^{19}$ Furthermore, most studies using clustering methods are based on predefined biomarkers that are often only loosely correlated with temporal changes in clinical symptoms or lung function. In addition, as opposed to atopy and inflammation, airway dynamics have been neglected as a characterising entity of asthma. ${ }^{20}$ Indeed, in asthma, both airway inflammation and variable airway obstruction are key components of this chronic disease. While airway inflammation and airway obstruction are often related, there are also mechanisms leading to lung functional abnormalities and asthma symptoms partly independent of airway inflammation but related to genetic factors, obesity, airway structure, and shear stress phenomena. ${ }^{20-22}$ 
There is recent evidence of daily fluctuations in inflammatory markers such as eosinophil counts, ${ }^{23}$ exhaled nitric oxide ${ }^{24}$ and, in particular, lung function. ${ }^{25}$ The fluctuation behaviour of lung function contains unexpected amounts of information on asthma characteristics such as airway obstruction and may provide more accurate clues regarding phenotype stability. ${ }^{2025-27}$ Furthermore, time series of daily lung function measurements display non-random fluctuations and long-range correlation properties significantly related to asthma phenotype ${ }^{26}$ and to exacerbation risk. ${ }^{25}$ In addition, these seem to have predictive power regarding clinical treatment response, for example, to long-acting $\beta$-agonists. ${ }^{28}$

Recent data suggest that in some asthma phenotypes, step-up treatment with long-acting $\beta$-agonists is more effective than an increase in anti-inflammatory treatment. ${ }^{29}$ The results shown in ref. ${ }^{28}$ thus suggest that airway function and its interaction with environmental stimuli may characterise a specific asthma phenotype, which could potentially benefit from different therapeutic approaches. ${ }^{29}$

We hypothesised that we could identify such subgroups of asthmatic participants with specific airway functional response to their time-varying environmental stimuli by investigating the patterns of fluctuation in airway function. Thus, time series fluctuation analysis and clustering methods could be combined, resulting in a novel, data-driven method for lung functional asthma phenotyping. We call this approach fluctuation-based clustering (FBC). It provides a new, complementary dimension for observer-independent asthma phenotyping.

Herein, we aim to determine whether FBC is able to distinguish children with asthma from healthy children. Furthermore, we tested whether different lung functional phenotypes existed within the groups of individuals with asthma and healthy individuals, and whether they were associated with predefined clinical asthma phenotypes, environmental factors, as well as genetic factors and inflammatory biomarkers of asthma. We performed this study prospectively in the Protection Against Allergy - Study in Rural Environments (PASTURE)/EFRAIM cohort of asthmatic and healthy children, ${ }^{30-32}$ in which serial measurements of twice-daily lung function parameters were obtained within a time window of four consecutive weeks.

The second dataset consisted of twice-daily lung function parameters measured over an entire year in a cohort of mildly/ moderately and therapy-resistant, severely asthmatic adults (Pan-European Longitudinal Assessment of Clinical Course and BIOmarkers in Severe Chronic AIRway Disease (BIOAIR) cohort). ${ }^{33}$ In this second independent dataset, we tested whether FBC could discriminate individuals with mild-to-moderate asthma from individuals with severe asthma. This aim was motivated by our previous observations, ${ }^{26}$ namely, that severe asthmatics show different lung function fluctuation patterns when compared with individuals with mild-to-moderate asthma, despite large variation and overlap of mean lung function between these two groups. The analysis of the second cohort was mainly done for the purpose of method validation using an additional independent data set and not with the intention of comparing the two cohorts directly.

\section{METHODS}

Our method, comprehensively described in the online supplementary material (OSM), aims to group or cluster individuals with similar fluctuation patterns in their lung function. To this end, we took into account both the mean lung function during the window of observation and also the magnitude and frequency of the fluctuations around the mean. This was accomplished by looking at each patient's entire empirical distribution of lung function parameters obtained during the time window of measurement. However, in order to more easily deal with missing measurements, our method does not take into account the chronological order of the measurements. Thereby, the temporal dimension is neglected.

Our cluster construction procedure does not make use of any further clinical parameters but is solely based on either of the two lung function parameters, peak expiratory flow (PEF) and forced expiratory volume during the first second $\left(\mathrm{FEV}_{1}\right)$. In order to test whether the clusters obtained via $\mathrm{FBC}$ indeed grouped together participants who shared meaningful clinical and diagnostic features, we compared the predominant clinical characteristics of patients in the clusters found in the cohort of children with previously published clinical symptoms and asthma phenotypes,${ }^{15}$ in particular, with respect to atopic, inflammatory (fraction of exhaled nitric oxide (FeNO)) and genetic markers. Furthermore, in the second cohort of asthmatic adults, we tested whether the clusters found discriminated mild-to-moderate asthma from severe therapy-resistant asthma.

\section{Study design}

The current proof of concept study has been prospectively embedded in the PASTURE/EFRAIM cohort. This is a prospective birth cohort study of children from rural areas in five European countries. Its design has been described elsewhere. ${ }^{30} 3134$

Furthermore, we also illustrated our methodology using data from the BIOAIR study (ClinicalTrials.gov identifier: NCT00555607). This study was designed to characterise the course of severe chronic airway diseases over time using a multitude of different clinical outcomes. The design has been described in detail elsewhere. ${ }^{33}$ Both studies were approved by all ethics committees of the corresponding participating study centres.

\section{Study population and definition of clinical phenotypes}

Within the PASTURE/EFRAIM cohort, at the age of 6 years, $\mathrm{n}=799$ of $\mathrm{n}=1133$ asthmatic and healthy children were enrolled. A percentage of 51.7 of the children were born in a farming environment. About $8.4 \%$ showed symptoms of asthma according to standardised assessments of clinical symptoms. ${ }^{30}$

Within the BIOAIR study, 169 adults with asthma were screened and classified as either severe asthmatics $(n=93)$ or mild-to-moderate asthmatics $(n=76) .{ }^{35}$ Patients were followed for 12 months with control visits at 4 monthly intervals. The present analysis included 138 patients (76 with severe asthma and 62 with mild-to-moderate asthma).

See tables in the OSM for more details.

\section{Lung function measurements}

See Methods, and part 1.2 of OSM for details. Within the PASTURE/EFRAIM cohort, measurements of PEF in $\mathrm{L} / \mathrm{min}$ and of $\mathrm{FEV}_{1}$ in $\mathrm{L}$ were recorded over a 4-week period at study participants' homes twice-daily (morning and evening). Ideally, this resulted in a total of 56 home measurements per study participant.

Within the BIOAIR study, lung function (PEF in $\mathrm{L} / \mathrm{min}$ and $\mathrm{FEV}_{1}$ in $\mathrm{L}$ ) was recorded twice-daily using electronic diaries over a period of 12 consecutive months. Ideally, this resulted in a total of 730 measurements per study participant.

Measured values of PEF and $\mathrm{FEV}_{1}$ were standardised using recently published reference data for spirometry. ${ }^{36}$ 
A

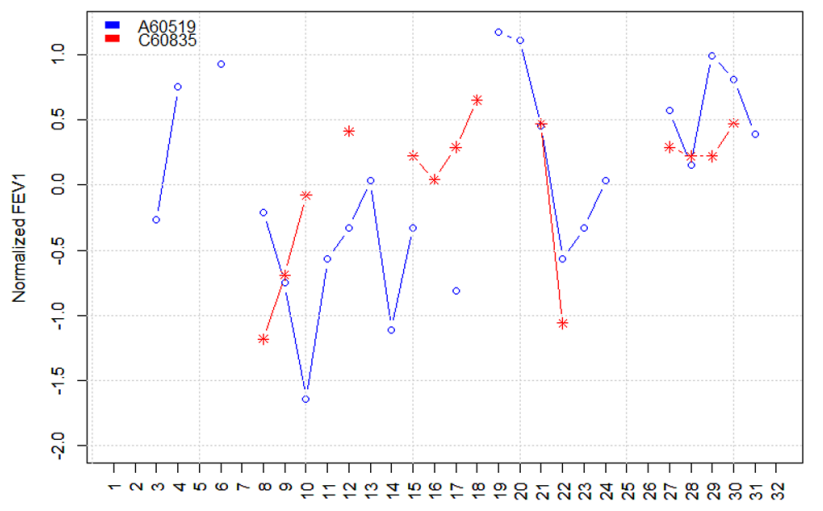

Day

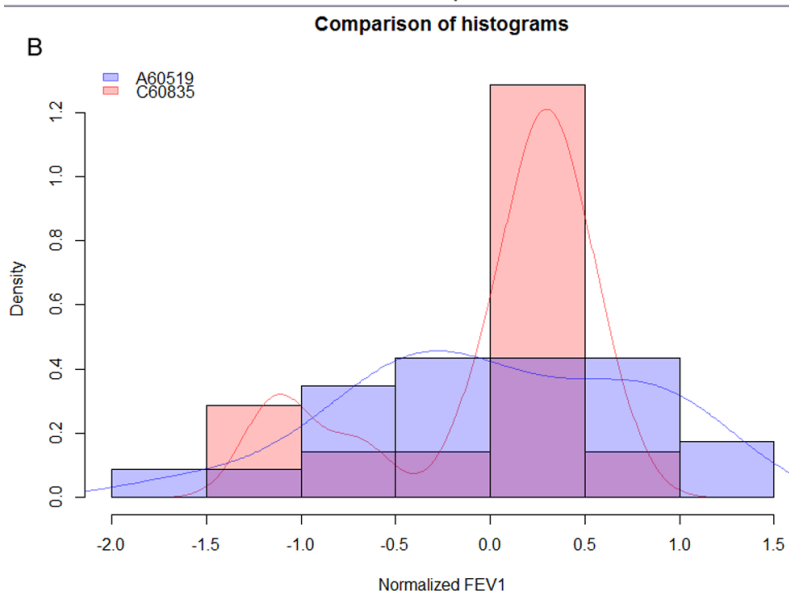

Figure $1 \mathrm{FEV}$, values (normalised as described in subsection 2.1 of the online supplementary material) collected in the mornings by two participants during a time window of 4 weeks (PASTURE/EFRAIM cohort). (A) displays the values as time series. Marked differences in the pattern of missing values and in the total length of the time series become apparent. Panel (B) displays the values as histograms, disregarding the chronological order of the data. This representation reveals that both time series share a common mean value. However, the data are distributed very differently, displaying completely different fluctuation patterns. Such differences can be quantified by descriptors such as variance, skewness, kurtosis and higher moments of the distribution. PASTURE, Protection Against Allergy - Study in Rural Environments.

\section{Brief overview of the computational methodology}

Two examples from the PASTURE/EFRAIM cohort of morning $\mathrm{FEV}_{1}$ time series are shown in figure $1 \mathrm{~A}$. While the mean values of both time series may be nearly the same, important and clinically relevant differences are reflected in the amplitude and frequency of fluctuations around the mean. Such differences become apparent by looking at the distribution of values (figure 1B).

Our method comprises five main steps: (1) First, after quality control, we select a high-quality 'seeding' data subset we refer to as the gold standard. The distribution of lung function measurements of a given participant is compared with the distributions of all the other participants. This pair-wise comparison is done using the Earth Mover's Distance (see OSM). These comparisons yield a collection of distance values for each participant in the cohort, which characterises every participant. We call these collections 'lung function profiles'. (2) We perform an initial agglomerative hierarchical clustering of the gold standard based

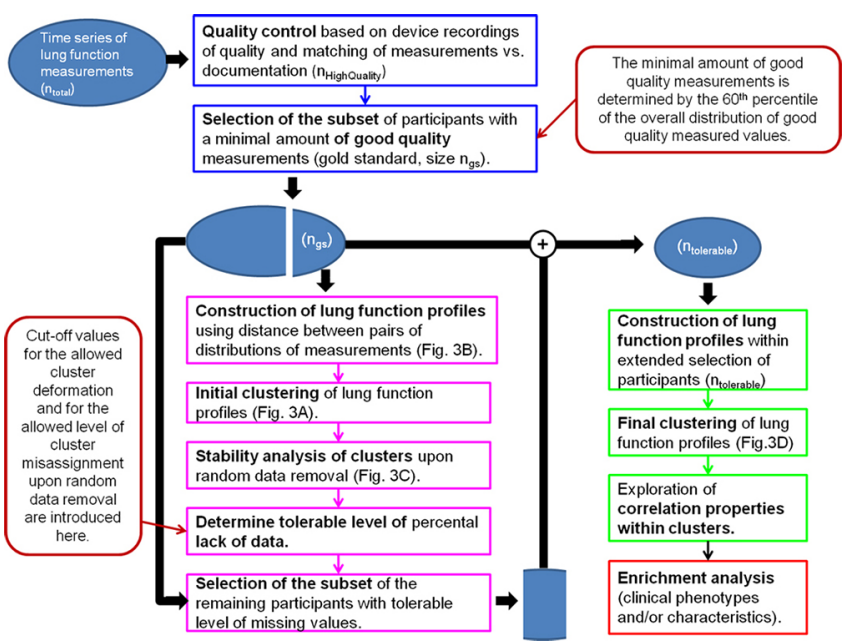

Figure 2 Workflow of the method exemplified based on data from the PASTURE/EFRAIM cohort. Blue rectangles: Based on quality and compliance criteria (see Methods section, part 2.1 of online supplementary material), we select a subset of participants with a nearly complete collection of high-quality measurements. This subset is used as our gold standard (size $n_{\mathrm{gs}}$ ). Pink rectangles: within the gold standard, we calculate the matrix of pairwise distances between all patients' distributions of lung function measurements. Each row within this matrix constitutes what we call the lung function profile of each patient within the given cohort. We use these profiles as the phenotypic fingerprint of each of these patients within the context of the cohort and perform hierarchical clustering on the rows of the matrix (see also figure 3, panels $A$ and $B$ ). On the resulting dendrogram, we then identify potentially suitable clusters (see also figure 3 , panel A). In order to include those participants who had more missing values, we analyse the stability of the clusters upon percental data removal (see also figure 3, panel C). This step is what helps determine the maximal number of missing values allowable and enables us to extend the gold standard to a possibly larger subset of the cohort (size $n_{\text {tolerable }}$ ). Green rectangles: for this extended selection of participants, the aforementioned matrix is again calculated, and a final dendrogram is obtained on the basis of hierarchical clustering of the matrix rows (see also figure 3, panel D). Red rectangle: we evaluate the method by testing whether the clusters found are significantly enriched in clinical phenotypes or characteristics of interest. More details on each step can be found in the Methods section and in the OSM. PASTURE, Protection Against Allergy - Study in Rural Environments.

on the Euclidean distance between the participants' lung function profiles. Subsequently, we determine, in a data-driven manner, the tolerable levels of missing values by means of analysing the stability of the clustering of the gold standard upon random data removal. (3) We extend the gold standard by including those participants not exceeding the tolerable levels of missing values. (4) We perform the final clustering of participants, and (5) we evaluate the method by testing whether patients within a given cluster exhibit specific clinical characteristics. This analysis of the resulting clusters, which uses information that was not used to inform the clustering procedure, yields criteria for selecting the number of clusters. (See figure 2 for a schematic representation of the method exemplified on data from the PASTURE/ EFRAIM cohort, and figure 3 for steps 1-4.)

Within the PASTURE/EFRAIM cohort, the analysis was conducted using the morning $\mathrm{FEV}_{1}$ measurements for the following reasons: evening measurements are more influenced by various daytime environmental stimuli than morning 
A

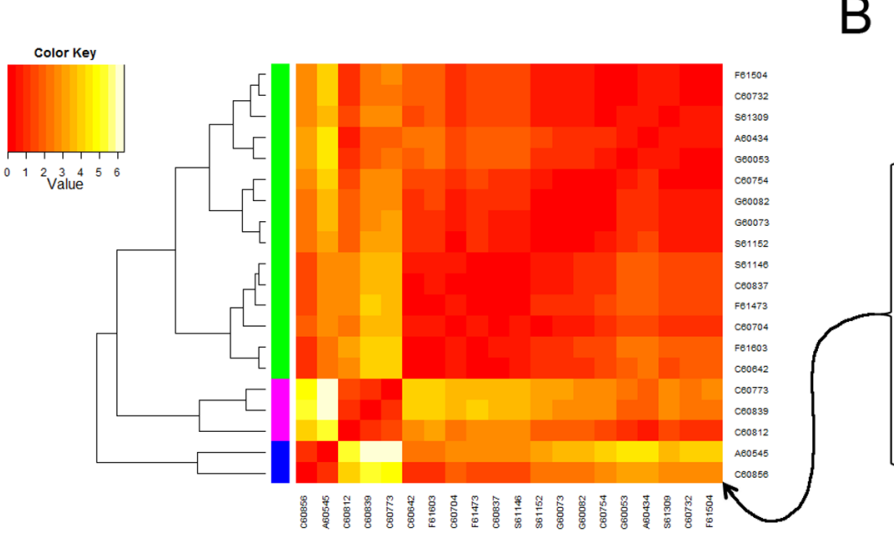

C

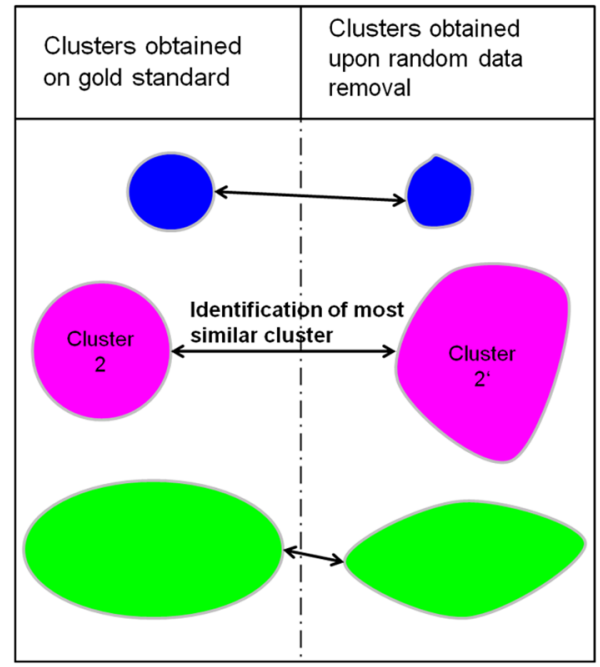

B

Participant $\mathbf{C 6 0 8 5 6}$

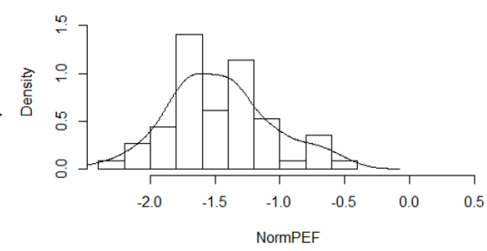

Participant F61504

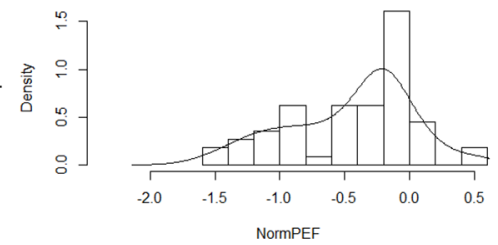

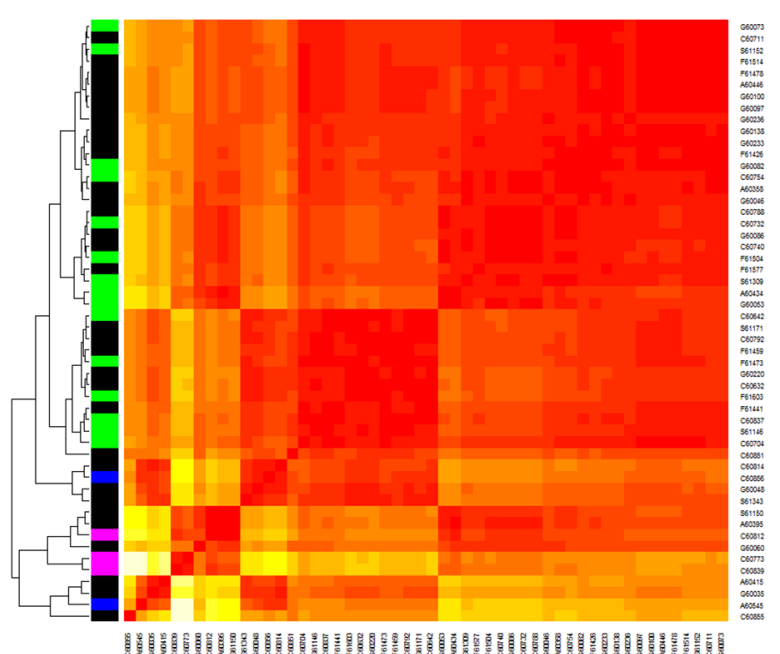

Figure 3 Workflow of the method, steps 1-4. (A) Heatmap representation of the matrix of lung function profiles. To the left of the matrix: dendrogram resulting from hierarchical clustering. Each row in the matrix corresponds to one participant in the gold standard, a subset of the cohort selected thorough quality and compliance criteria. Each entry in the matrix is the result of a distance calculation between a pair of distributions of lung function measurements (B). Three identified clusters are marked with colour bars (green, blue and magenta) for illustration purposes. (C) Assessment of the stability of the clusters upon random removal of a fixed percentage of measurements. Stability requirements allow for the calculation of a tolerable percentage of missing data. The gold standard subset is extended by including participants with a tolerable level of missing measurements. A new matrix of lung function profiles is constructed, and hierarchical clustering on those profiles is performed using this extended data set (D). Cluster assignment of previous cluster members is visible via colour bars on black background. The data underlying all panels are hypothetical normalised PEF values created/selected to illustrate the method. PEF, peak expiratory flow.

measurements. Furthermore, during the measurement in children, PEF may still be slightly more dependent on cooperation than $\mathrm{FEV}_{1}$. Lastly, in many randomised controlled trials, $\mathrm{FEV}_{1}$ is still the most often used outcome parameter. The results obtained using other combinations of lung function parameters $\left(\mathrm{PEF}\right.$ or $\mathrm{FEV}_{1}$ ) and time slots (morning, evening and entire day) can be found in the OSM for the sake of illustration.

\section{RESULTS}

In the application and evaluation of our method, the results are generated according to the FBC methodology, as depicted in the FBC workflow (figure 2). All five steps of this workflow (as described above in the Methods section) were applied to the data from the PASTURE/EFRAIM cohort. In order to include participants with more missing values (step 3), we analysed the stability of the clusters found in the gold standard upon random removal of different percentages of measurements. Using suitable tolerance levels of cluster disruption, we were able to include participants in the analysis that had $20 \%$ of their values missing.

The final clustering together with each participant's individual collection of normalised morning $\mathrm{FEV}_{1}$ values are depicted in figure 4.

The statistical analysis and characterisation of the clusters found is presented in full detail below. The very last subsection of the results section is devoted to the statistical analysis and characterisation of the clusters found in the BIOAIR cohort. There, we carried out the analysis only on the gold standard and using whole day measurements of $\mathrm{FEV}_{1}$.

\section{Enrichment analysis and clinical characterisation of clusters identified in the PASTURE/EFRAIM cohort}

We investigated whether the clusters found represent asthma-specific clinical characteristics and phenotypes. Moreover, we looked for potential functional differences among 

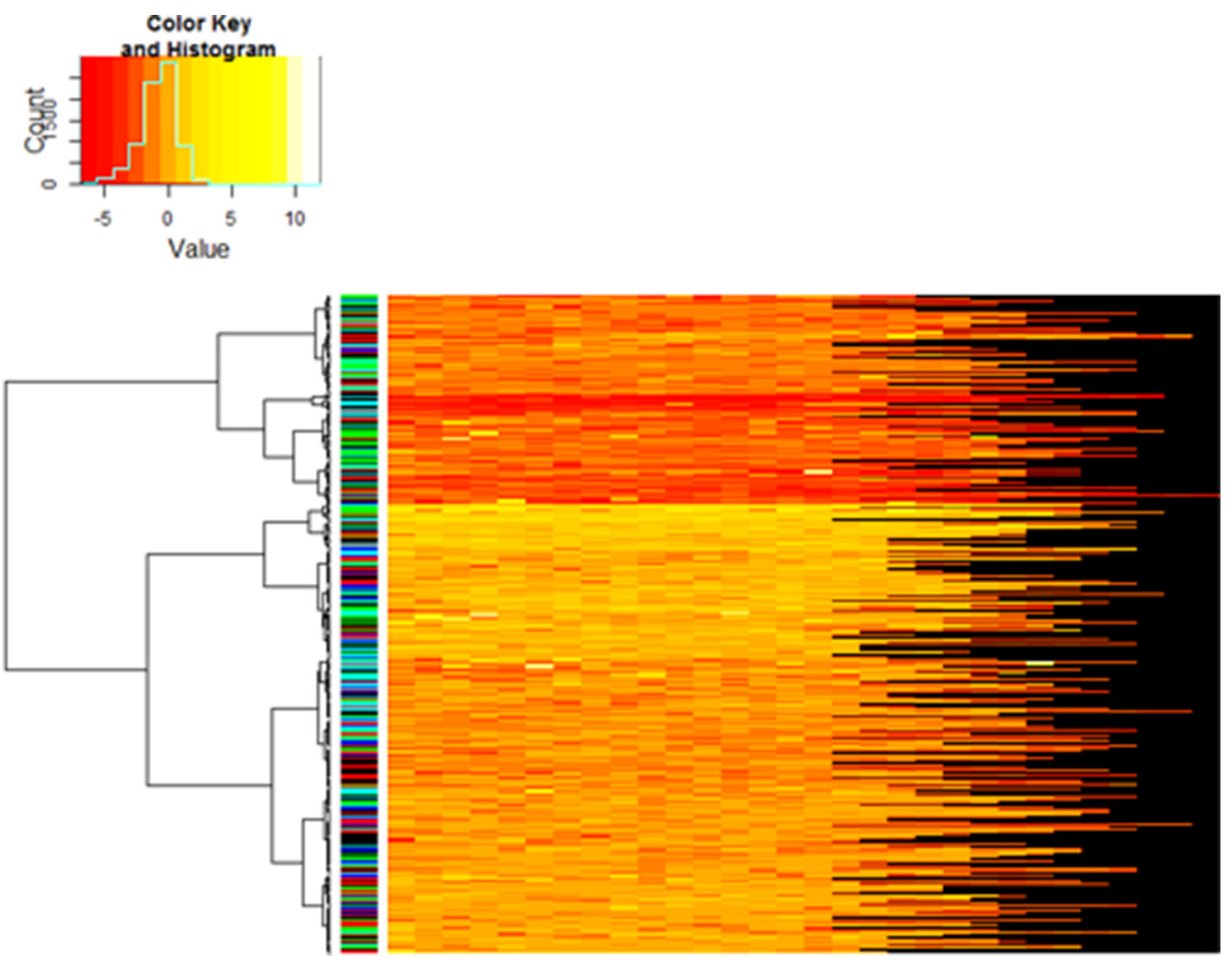

Figure 4 Dendrogram obtained via hierarchical clustering of each participant's lung function profile within the subset obtained after extension of the gold standard (PASTURE/EFRAIM cohort). The heatmap does not show the participants' lung function profiles. Instead, each row corresponds to one participant and illustrates their individual collection of normalised morning FEV values. The different lengths of the rows reflect the aforementioned compliance issues within this cohort. The colour bar between the dendrogram and the heatmap indicates the colour-coded country of the participants. The random pattern of colours demonstrates that the individual clusters are not enriched in any particular nationality (verified by hypergeometric test, results not shown). PASTURE, Protection Against Allergy - Study in Rural Environments.

the healthy participants. To this end, we determined whether the individual clusters were significantly enriched (see Methods and part 2.4 of the OSM) in the following clinical phenotypes and characteristics available within the cohort: children of farmers versus those of non-farmers, gender, atopic disease, asthma diagnosis, recurrent wheeze, episodic wheeze, atopic asthma, non-atopic asthma, presence of the risk allele containing the SNP rs7216389 in the gasdermin B (GSDMB) coding region of chromosome $17 \mathrm{q} 21$ (see, eg, ref ${ }^{37}$ ), FeNO as a marker of airway inflammation, and significant bronchodilator response (see online supplementary table E1). Results and statistical significance are reported in table 1. Results for all variables, time slots and clustering multiplicities can be found in online supplementary tables S2-S7.

In cluster 1 , the mean morning $\mathrm{FEV}_{1}$ measurements tended to be lower, which is consistent with more airway obstruction. This cluster also displayed the lowest mean coefficient of variation in $\mathrm{FEV}_{1}$ values, although the highest SD.

The enrichment analysis unveiled significantly more children with clinical symptoms of asthma in clusters 1 and 2 . In cluster 1 , more children with asthma, according to the broader definition, lower lung function and positive bronchodilator response but no allergic predisposition, were found than expected by chance. Cluster 2 was significantly enriched in children with recurrent wheezing symptoms and who used inhaled corticosteroids as asthma treatment. In contrast to cluster 1, cluster 2 is characterised by the highest mean variability in $\mathrm{FEV}_{1}$, which is almost five times higher than in cluster 1 . Thus, FBC was helpful in identifying two lung functional phenotypes, each of which contributing differently to the clinical manifestation of asthma. These are namely functionally different in terms of mean, coefficient of variation $(\mathrm{CV})$ of daily lung function and in terms of bronchodilator responsiveness, but not in terms of atopy or inflammatory markers (FeNO).

Clusters 3 and 4 appeared different and showed the highest proportion of healthy participants $(>80 \%)$. Interestingly, FBC similarly separated two functional phenotypes in the healthy control participants (clusters 3 and 4) with lung function values typically in the normal range. Cluster 3 showed a group low mean $\mathrm{FEV}_{1}$ and low variability and was enriched with individuals living in a farming environment. There were significantly more participants in cluster 3 (79.6\%) who carried the risk allele GSDMB rs7216389. Cluster 4 contained predominantly healthy children-and more girls than boys - with high normalised mean lung function and high mean variability in $\mathrm{FEV}_{1}$. This cluster was depleted (ie, fewer than expected by chance) of participants carrying the risk allele GSDMB rs7216389.

Clusters 1 and 3 appeared similar with both showing less variable $\mathrm{FEV}_{1}$ and a comparatively low mean $\mathrm{FEV}_{1}$. At the same time, cluster 1 was clearly dominated by children with clinical symptoms of asthma, whereas cluster 3 corresponded to a healthy 
Table 1 Absolute (and relative) number of individuals with the phenotype or characteristic (see online supplementary table E1 for definitions) specified in the first column in the different clusters. Enrichment in a given phenotype/characteristic is marked in bold letters. Depletion in a given phenotype/characteristic is marked in italics

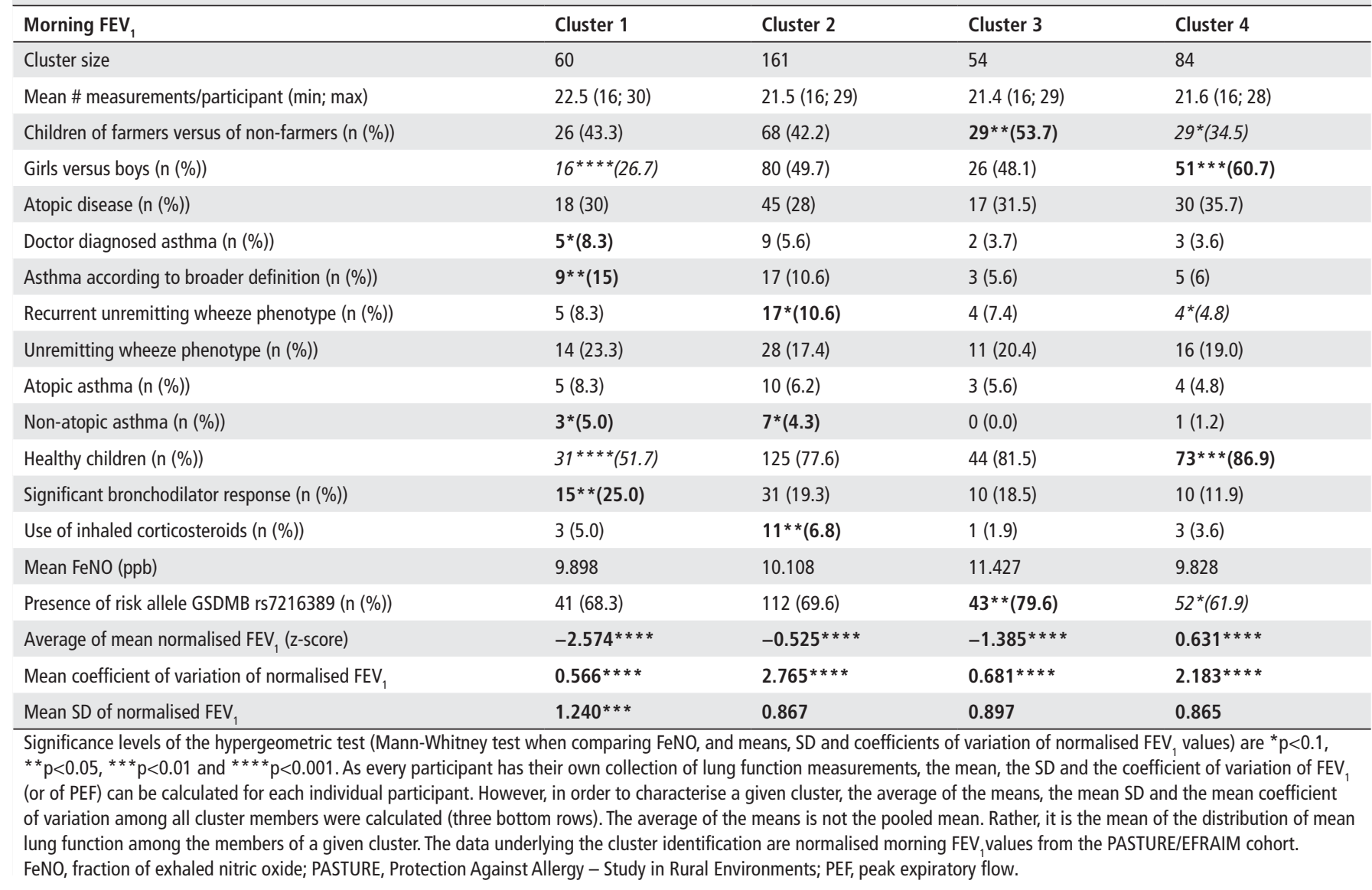

subgroup. Thus, the same 'functional type' found in individuals with asthma can be found to a milder degree in healthy children. Moreover, the four clusters were not significantly different in terms of the levels of FeNO, and no cluster was enriched in atopic individuals.

\section{Independent application using dataset of mild-to-moderate and therapy-resistant severe adult asthmatics (BIOAIR cohort)}

In order to explore the ability of our method to discriminate between different levels of disease severity, we analysed data from 138 patients consisting of 76 clinically defined therapy-resistant severe asthmatics and 62 mild-to-moderate asthmatics. ${ }^{33}$ According to the same criterion defined above, we identified the gold standard, which consisted of 45 patients. We then conducted hierarchical clustering on the lung function profiles of these patients and cut the dendrogram at the height that yielded two clusters. By means of enrichment analysis, we determined that these two clusters separated the gold standard into mainly mild-to-moderate asthmatics, on the one hand, and predominantly severe asthmatics, on the other hand (table 2).

This is particularly interesting since the group mean of $\mathrm{FEV}_{1}$ in the clinically defined mild-to-moderate asthmatics (median $=-2.0$, Interquartile interval $=(-2.4 ;-1.4)$ ) showed a large overlap with the group of severe asthmatics $(-2.8$ $(-3.5 ;-1.6))$. Consequently, a classifier solely based on a threshold value of mean lung function would frequently yield a misclassification. Using the patients' individual fluctuation patterns as an additional discriminative feature may mitigate this issue.

\section{DISCUSSION}

\section{Main findings}

Both airway inflammation and reversible airway obstruction are key features of bronchial asthma in children and adults. Most published clustering methods for phenotyping asthma have used clinical, inflammatory or lung function markers at a single point in time. However, for the clinician in daily practice, the history of the day-to-day obstructive response of the airways to external stimuli in the patient's environmental context is crucial in diagnosis and treatment decisions. So far, temporal fluctuation of daily lung function has never been used to functionally phenotype or cluster groups of patients with asthma. By combining fluctuation analysis and clustering methods, we present here a novel clustering approach to lung functional phenotyping (FBC). Moreover, we have developed a procedure that effectively determines the tolerable number of missing measurements in a datadriven manner.

Using FBC in the cohort of children, we identified two distinct lung functional phenotypes of participants with well-known clinical characteristics of asthma. These were significantly different from two functional clusters consisting of mainly healthy infants. Mostly non-atopic asthmatics were over-represented in clusters 1 and 2. Children in the asthma-related clusters 1 and 2 were different in their degree of obstruction, bronchodilator responsiveness and medication use. While we found a predominance of 
Table 2 Absolute (and relative) number of individuals with the phenotype or characteristic specified in the first column in the different clusters. Enrichment (hypergeometric test) in a given phenotype/ characteristic is marked in bold letters

\begin{tabular}{|c|c|c|}
\hline & Cluster 1 & Cluster 2 \\
\hline Number of patients & 28 & 17 \\
\hline $\begin{array}{l}\text { Mean \# measurements/participant } \\
\text { (min; max) }\end{array}$ & $575(477 ; 708)$ & $574(481 ; 706)$ \\
\hline \multicolumn{3}{|l|}{ Airway disease } \\
\hline Mild-to-moderate asthma (n (\%)) & $17(60.7)^{* * *}$ & $5(29.4)$ \\
\hline Severe asthma (n (\%)) & $11(39.3)$ & $12(70.6)^{* * *}$ \\
\hline $\begin{array}{l}\text { Average of mean normalised FEV } \\
\text { (z-score) }\end{array}$ & $-1.7^{* \star * *}$ & $-3.6^{* * * *}$ \\
\hline $\begin{array}{l}\text { Mean coefficient of variation of } \\
\text { normalised FEV }\end{array}$ & $0.430 * * * *$ & $0.217^{* * * *}$ \\
\hline Mean SD of normalised FEV & 0.5 & 0.6 \\
\hline
\end{tabular}

Significance levels of the hypergeometric test (Mann-Whitney test when comparing means, SD and coefficients of variation of normalised FEV , values) are ${ }^{*} \mathrm{p}<0.1$, ${ }^{* *} p<0.05,{ }^{* * *} p<0.01$ and ${ }^{* * * *} p<0.001$. As every participant has their own collection of lung function measurements, the mean, the SD and the coefficient of variation of $\mathrm{FEV}_{1}$ (or of PEF) can be calculated for each individual participant. However, in order to characterise a given cluster, the average of the means, the mean SD and the mean coefficient of variation among all cluster members were calculated (three bottom rows). The average of the means is not the pooled mean. Rather, it is the mean of the distribution of mean lung function among the members of a given cluster. The data underlying the cluster identification are normalised

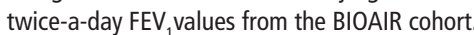

BIOAIR, Pan-European Longitudinal Assessment of Clinical Course and BIOmarkers in Severe Chronic AIRway Disease.

children with good $\beta_{2}$ response in cluster 1 , more children were treated with inhaled corticosteroids in cluster 2. Interestingly, in cluster 1, we found asthmatic children with low lung function, low variability and significant bronchodilator response, whereas in cluster 2, mean $\mathrm{FEV}_{1}$ was higher and accompanied by high daily variability.

Similarly, in the predominantly healthy clusters (3 and 4), cluster 3 was characterised by low-normal mean $\mathrm{FEV}_{1}$ and low variability, whereas 4 showed high mean $\mathrm{FEV}_{1}$, higher natural fluctuations of $\mathrm{FEV}_{1}$ and a predominance of girls. In fact, we found the functional phenotypes characterised by low mean and low variability, as well as the functional phenotypes characterised by high mean and high variability in asthma, but also to a milder degree in predominantly healthy children (clusters 1 and 3, and clusters 2 and 4, respectively). Since inflammatory markers (atopic predisposition and FeNO) were not different between these four clusters, our data suggest that FBC identifies dynamic lung functional characteristics of the airways in response to the given environment, at least partially independent of their atopic and inflammatory status.

Our data also suggest that intrinsic hereditary and interacting environmental factors may contribute to asthma and, to a milder degree, to healthy phenotypes. Hereditary effects, such as sex effects (clusters 1 and 4), and also associations with asthma-related genes $(17 \mathrm{q} 21)$ were observed in clusters 3 and 4. In cluster 3 , we found an enrichment of children carrying the risk allele containing the SNP rs7216389 in the gasdermin $\mathrm{B}(G S D M B)$ coding region of chromosome $17 \mathrm{q} 21$, and in cluster 4 , a depletion of such individuals. The presence of this allele has been linked to asthma, exacerbation risk and changes in $\mathrm{FEV}_{1}$. In particular, specific associations with bronchial hyper-responsiveness, severe asthma and asthma that is poorly controlled by current medications have been found (see ref ${ }^{37}$ and the citations therein). The association of rs7216389 with exacerbation risk, bronchial responsiveness ${ }^{38}$ and also gene interactions with arginase1 (rs37756780, ARG1) ${ }^{39}$ may be a potential underlying mechanism to explain the enrichment and depletion of SNP rs7216389 in clusters 3 and 4, respectively. Moreover, recently, a gene-by-environment interaction of locus $17 \mathrm{q} 21$ has been demonstrated. ${ }^{40}$ In particular, it has been shown that individuals carrying the GSDMB rs7216389 variant particularly benefit from growing up in a farming environment due to its protective effect against virus-induced wheeze and non-atopic asthma. In cluster 3, children living in a farming environment were predominant. Their vulnerability resulting from carrying the risk allele GSDMB rs7216389 is thus compensated via the aforementioned protective effect. This may explain why the comparatively reduced lung function that is strikingly characteristic of the children in cluster 3 generally remains asymptomatic. Nevertheless, temporal variations of environmental triggers ${ }^{41}$ in the farming environment may particularly affect daily lung function.

Our results indicate that our methodology may be useful for phenotyping in existing asthma and also for functional phenotyping in healthy individuals. For instance, our findings regarding cluster 3 suggest that this cluster could potentially contain a subgroup of individuals at risk of developing asthma. Future longitudinal studies will be required in order to investigate this.

In our second cohort (BIOAIR), we found that our FBC method was able to discriminate between mild-to-moderate asthma and therapy-resistant severe asthma. Our findings are consistent with previous observations that fluctuation patterns in severe asthma are different than in mild asthmatics. ${ }^{26}$

Our method is based on distribution but not correlation properties of the lung function measurements. Calculating correlation from data with missing data points is prone to errors. Thus, while neglecting the time dimension, we gain robustness with respect to missing data, which increases the feasibility and clinical applicability of the method.

Limitations and advantages of the FBC method, especially in comparison with other existing clustering approaches to disease phenotyping, are discussed in the OSM.

\section{Clinical implications of this novel approach}

FBC identifies two distinct clusters of asthma patients and two clusters of healthy participants with specific dynamic lung functional interaction patterns to the stimuli in their given environment, which is at least partly independent of the inflammatory or atopic status, but may be related to hereditary (sex, $17 \mathrm{q} 21$ $(G S D M B))$ and environmental factors (eg, farming). We believe that lung functional stability over an extended window of time is an additional and important characteristic to be considered in asthma research and is different from a single-point-in-time characterisation. This is particularly important since it becomes increasingly evident that many asthma biomarkers need to be interpreted within the context of the patient's given environment. We hypothesise that these dynamic lung functional characteristics are relevant for the understanding of disease stability over time and also for the search for treatable traits.

There is increasing evidence that the causal relation between airway inflammation and subsequent airway obstruction is weak and complex. Lung functional impairment, smooth muscle mechanics ${ }^{21}$ and impaired elastic recoil of the airways in obese patients ${ }^{42}$ can contribute to airway obstruction and asthma symptoms independent of airway inflammation. ${ }^{8}$ As demonstrated in the BADGER study, ${ }^{29}$ some patients may benefit more from bronchodilator therapy than from step-up anti-inflammatory 
treatments in order to achieve asthma stability. FBC might help identify such patients. Future studies will need to demonstrate whether FBC phenotyping (eg, cluster 1) may contribute to this clinical decision making. Previous work has already shown that lung function fluctuation patterns can predict treatment response to long-acting $\beta$-agonists. ${ }^{28}$ Within the context of adult asthma, the method contributes to the identification of severe therapy-resistant asthmatic patients, a known distinct entity, which is typified by a lack of asthma control despite appropriate anti-inflammatory treatment. In these patients, not only steroid resistance, but also airway remodelling and fixed functional airway impairment contribute to poor asthma control. In such cases, FBC could help identify patients with lung mechanical impairment. FBC does not replace other clustering methods but as a complementary method could provide evidence of mechanical impairment by way of measurements taken in a telemonitoring setting.

\author{
Author affiliations \\ 'University Children's Hospital (UKBB), University of Basel, Basel, Switzerland \\ ${ }^{2}$ Endothelial Cell Biology Unit and Department of Applied Mathematics, School of \\ Molecular \& Cellular Biology, School of Mathematics, University of Leeds, Leeds, UK \\ ${ }^{3}$ Dr von Hauner Children's Hospital, Ludwig Maximilians University, Member of the \\ German Center for Lung Research (DZL), Munchen, Germany \\ ${ }^{4}$ Department of Paediatric Respiratory Medicine, Inselspital, University Children's \\ Hospital of Bern, University of Bern, Bern, Switzerland \\ ${ }^{5}$ Department of Health Protection, National Institute for Health and Welfare, Kuopio, \\ Finland \\ ${ }^{6}$ Department of Public Health, University of Helsinki, Helsinki, Finland \\ ${ }^{7}$ Department of Respiratory Disease, University Hospital of Besançon, Besançon, \\ France \\ ${ }^{8}$ Children's Hospital, Schwarzach, Austria \\ ${ }^{9}$ Children's Hospital of Eastern Switzerland, St. Gallen, Switzerland \\ ${ }^{10}$ Department of Pediatric Pneumology and Allergy Campus St. Hedwig, KUNO \\ Children's University Hospital, Regensburg, Germany \\ ${ }^{11}$ Clinic for Pediatric Pneumology and Neonatology, Hannover Medical School, \\ Hannover, Germany \\ ${ }^{12}$ Experimental Asthma and Allergy Research Unit, The National Institute of \\ Environmental Medicine, Karolinska Institutet, Stockholm, Sweden \\ ${ }^{13}$ Department of Internal Medicine, Asthma and Allergy, Medical University of Lodz, \\ Lodz, Poland
}

Correction notice This article has been corrected since it was published Online First. The following changes have been made: 1) Sven-Erik Dahlen's name was corrected; 2) Affiliation 12 was corrected; 3) Reference 13 citation was incorrect, this has now been corrected; 4) Collaborators section has been corrected.

Acknowledgements The authors would like to thank Karine Landgren Hugentobler and Anna Longjaloux for proofreading the manuscript. The authors would also like to express their gratitude to PhD candidate Delphine Meier for carrying out the computations concerning the analysis of the BIOAIR cohort data.

Collaborators The members of the PASTURE study group are (in alphabetical order): Sondhja Bitter ${ }^{A, B}$, Charlotte Braun-Fahrländer ${ }^{A, B}$, Gisela Büchele ${ }^{C}$, MarieLaure Dalphin ${ }^{\mathrm{D}}$, Martin Depner ${ }^{\mathrm{H}}$, Gert Doekes ${ }^{\mathrm{E}}$, Markus J. Ege ${ }^{\mathrm{H}}$, Remo Frei ${ }^{\mathrm{F}}$, Jon Genuneit ${ }^{C}$, Maija-Riitta Hirvonen ${ }^{G}$, Anne Hyvärinen ${ }^{K}$, Anne M. Karvonen ${ }^{k}$, Vincent Kaulek ${ }^{D}$, Georg Loss ${ }^{A, B}, H$, Petra Ina Pfefferle ${ }^{N}$, Sami Remes', Harald Renz', Caroline Roduit ${ }^{\mathrm{O}}$, Marjut Roponen $^{\mathrm{G}}$, Bianca Schaub ${ }^{\mathrm{H}}$, Pekka Tiittanen ${ }^{\mathrm{K}}$, Juliane Weber ${ }^{H}$. ${ }^{A}$ Swiss Tropical and Public Health Institute, Basel, Switzerland. ${ }^{B}$ University of Basel, Basel, Switzerland. 'Institute of Epidemiology and Medical Biometry, University of Ulm, Ulm, Germany. ${ }^{D}$ Department of Respiratory Disease, UMR/CNRS 6249 chrono-environnement, University Hospital of Besançon, France. EUtrecht University, Institute for Risk Assessment Sciences (IRAS), Division of Environmental Epidemiology, Utrecht, Netherlands. FUniversity of Zurich, Children's Hospital, and Christine Kühne-Center for Allergy Research and Education, Zurich, Switzerland. ${ }^{6}$ Department of Environmental Science, University of Eastern Finland, Kuopio, Finland. "Dr von Hauner Children's Hospital, Ludwig Maximilians University, Munich, Germany; Member of the German Center for Lung Research (DZL). 'Department of Paediatrics, Kuopio University Hospital, Kopio, Finland. Institute for Laboratory Medicine and Pathobiochemistry, Molecular Diagnostics, Philipps University of Marburg, Marburg, Germany. ${ }^{K}$ Department of Health Protection, National Institute for Health and Welfare, Kuopio, Finland. NInstitute for Laboratory Medicine and Pathobiochemistry, Molecular Diagnostics, Philipps University of Marburg, Marburg, Germany. ${ }^{\circ}$ Children's Hospital, University of Zurich, and Christine Kühne-Center for
Allergy Research and Education, Zurich, Switzerland. The members of the BIOAIR study group are: Dr Roelinde Middelveld, Karolinska Institutet, Stockholm, Sweden. Professor Barbro Dahlén, Karolinska Institutet, Stockholm, Sweden. Dr Mina Gaga, University of Athens, Greece. Professor Nikos M. Siafakas, University of Crete, Greece. Professor Alberto Papi, University of Ferrara, Italy. Leonardo M. Fabbri, University of Modena, Italy. Professor Guy Joos, University of Gent, Belgium. Professor Klaus F. Rabe, Christian Albrechts University Kiel, Germany. Professor Sebastian L. Johnston, The Imperial College of Science and Technology, London, UK. Professor Pascal Chanez, University of Marseilles, France. Dr Mark Gjormarkaj, Italian Research Council, Palermo, Italy. Dr Peter H. Howarth, University of Southampton, UK. Professor Ewa Niżankowska-Mogilnicka, The Jagellonian University, Krakow, Poland.

Contributors ED-E, OF, JP, J-CD, JR, RL, EvM and UF planned the study; ED-E, NK, OF and the PASTURE Study Group were involved in the acquisition, management and interpretation of data; ED-E conceived the computational methodology and the algorithms, selected statistical tests to be used and implemented algorithms; NK conceived and implemented algorithms and performed data processing; MK planned genetic analyses and interpreted genetic data. ED-E, NK and OF performed statistical analyses; MK, S-ED and the BIOAIR Study Group were responsible for the study design, cohort collection and data analysis of the BIOAIR Study; ED-E, OF, EvM and UF wrote the manuscript; all authors provided substantial revisions and approval of the final manuscript.

Funding The PASTURE/EFRAIM study was supported by the European Commission research grants QLK4-CT-2001-00250, FOOD-CT-2006-31708 and KBBE-2007-2-206. OF (PASTURE/EFRAIM study) is the recipient of a Long-Term Research Fellowship by the European Respiratory Society (no. 675) and a training scholarship by the Austrian, German and Swiss Paediatric Respiratory Society. The BIOAIR study was supported by the following Swedish research funding bodies: The Medical Research Council, the Heart-Lung Foundation, the Vårdal Foundation, the Stockholm County Council (ALF), the Swedish Asthma and Allergy Association, the Swedish Foundation for Strategic Research, Konsul Th C Berghs Foundation, the Karolinska Institutet ScilifeLab collaborations on translational medicine (ChAMP project), the Innovative Medicines Initiative project U-BIOPRED (unbiased biomarkers for the prediction of respiratory disease outcomes) and Karolinska Institutet.

Competing interests None declared.

Ethics approval Ethics committees of the corresponding participating study centres.

Provenance and peer review Not commissioned; externally peer reviewed.

(c) Article author(s) (or their employer(s) unless otherwise stated in the text of the article) 2018. All rights reserved. No commercial use is permitted unless otherwise expressly granted.

\section{REFERENCES}

1 Corren J. Asthma phenotypes and endotypes: an evolving paradigm for classification. Discov Med 2013;15:243-9.

2 Kiley J, Smith R, Noel P. Asthma phenotypes. Curr Opin Pulm Med 2007;13:19-23.

3 Agache IO. From phenotypes to endotypes to asthma treatment. Curr Opin Allergy Clin Immunol 2013;13:249-56.

4 Lin TY, Poon AH, Hamid Q. Asthma phenotypes and endotypes. Curr Opin Pulm Med 2013;19:18-23.

5 Lötvall J, Akdis CA, Bacharier LB, et al. Asthma endotypes: a new approach to classification of disease entities within the asthma syndrome. J Allergy Clin Immunol 2011;127:355-60.

6 Poon AH, Hamid Q. Asthma endotypes: the right direction towards personalized medicine for asthma. Expert Rev Clin Immunol 2012;8:595-6.

7 Wenzel SE. Asthma phenotypes: the evolution from clinical to molecular approaches. Nat Med 2012;18:716-25.

8 Haldar P, Pavord ID, Shaw DE, et al. Cluster analysis and clinical asthma phenotypes. Am J Respir Crit Care Med 2008;178:218-24.

9 Prosperi MC, Sahiner UM, Belgrave D, et al. Challenges in identifying asthma subgroups using unsupervised statistical learning techniques. Am J Respir Crit Care Med 2013;188:1303-12.

10 Schatz M, Hsu JW, Zeiger RS, et al. Phenotypes determined by cluster analysis in severe or difficult-to-treat asthma. J Allergy Clin Immunol 2014;133.

11 Siroux V, Basagaña X, Boudier A, et al. Identifying adult asthma phenotypes using a clustering approach. Eur Respir J 2011;38:310-7.

12 Wu W, Bleecker E, Moore W, et al. Unsupervised phenotyping of Severe Asthma Research Program participants using expanded lung data. J Allergy Clin Immunol 2014;133:1280-8.

13 Fitzpatrick AM, Teague WG, Meyers DA, et al. Heterogeneity of severe asthma in childhood: confirmation by cluster analysis of children in the National Institutes of Health/National Heart, Lung, and Blood Institute Severe Asthma Research Program. J Allergy Clin Immunol 2011;127:382e1-389.

14 Just J, Gouvis-Echraghi R, Rouve S, et al. Two novel, severe asthma phenotypes identified during childhood using a clustering approach. Eur Respir J 2012;40:55-60. 
15 Moore WC. The natural history of asthma phenotypes identified by cluster analysis. Looking for chutes and ladders. Am J Respir Crit Care Med 2013;188:521-2.

16 Moore WC, Meyers DA, Wenzel SE, et al. Identification of asthma phenotypes using cluster analysis in the Severe Asthma Research Program. Am J Respir Crit Care Med 2010;181:315-23.

17 Smith JA, Drake R, Simpson A, et al. Dimensions of respiratory symptoms in preschool children: population-based birth cohort study. Am I Respir Crit Care Med 2008:177:1358-63.

18 Weatherall M, Travers J, Shirtcliffe PM, et al. Distinct clinical phenotypes of airways disease defined by cluster analysis. Eur Respir J 2009;34:812-8.

19 Boudier A, Curjuric I, Basagaña X, et al. Ten-year follow-up of cluster-based asthma phenotypes in adults. A pooled analysis of three cohorts. Am I Respir Crit Care Med 2013:188:550-60.

20 Stein RT, Holberg CJ, Morgan WJ, et al. Peak flow variability, methacholine responsiveness and atopy as markers for detecting different wheezing phenotypes in childhood. Thorax 1997; 52:946-52.

21 Tschumperlin DJ, Drazen JM. Chronic effects of mechanical force on airways. Annu Rev Physiol 2006;68:563-83.

22 Martinez FD, Vercelli D. Asthma. The Lancet 2013:382:1360-72.

23 Fleming L, Wilson N, Regamey N, et al. Use of sputum eosinophil counts to guide management in children with severe asthma. Thorax 2012;67:193-8.

24 Stern G, de Jongste J, van der Valk R, et al. Fluctuation phenotyping based on daily fraction of exhaled nitric oxide values in asthmatic children. J Allergy Clin Immunol 2011:128:293-300.

25 Frey U, Brodbeck T, Majumdar A, et al. Risk of severe asthma episodes predicted from fluctuation analysis of airway function. Nature 2005:438:667-70.

26 Thamrin C, Nydegger R, Stern G, et al. Associations between fluctuations in lung function and asthma control in two populations with differing asthma severity. Thorax 2011;66:1036-42.

27 Thamrin C, Zindel J, Nydegger $R$, et al. Predicting future risk of asthma exacerbations using individual conditional probabilities. J Allergy Clin Immuno 2011;127:1494-502.

28 Thamrin C, Stern G, Strippoli MP, et al. Fluctuation analysis of lung function as a predictor of long-term response to beta2-agonists. Eur Respir $J$ 2009;33:486-93.
29 Lemanske RF, Mauger DT, Sorkness CA, et al. Step-up therapy for children with uncontrolled asthma receiving inhaled corticosteroids. N Engl J Med 2010;362:975-85.

30 Depner M, Fuchs $\mathrm{O}$, Genuneit J, et al. Clinical and epidemiologic phenotypes of childhood asthma. Am J Respir Crit Care Med 2014:189:129-38.

31 Ege MJ, Herzum I, Büchele G, et al. Prenatal exposure to a farm environment modifies atopic sensitization at birth. J Allergy Clin Immunol 2008;122:407-12.

32 Delgado-Eckert E, Kumar N, Fuchs O, et al. Asthma phenotypes determined by a novel fluctuation based clustering method using a time window of lung function observations. Eur Respir J 2015;46(suppl 59):0A1473.

33 Kupczyk M, Haque S, Sterk PJ, et al. Detection of exacerbations in asthma based on electronic diary data: results from the 1-year prospective BIOAIR study. Thorax 2013;68:611-8.

34 von Mutius E, Schmid S, Group PS. The PASTURE project: EU support for the improvement of knowledge about risk factors and preventive factors for atopy in Europe. Allergy 2006;61:407-13.

35 Kupczyk M, Haque S, Middelveld RJ, et al. Phenotypic predictors of response to oral glucocorticosteroids in severe asthma. Respir Med 2013;107:1521-30.

36 Stanojevic S, Wade A, Stocks J, et al. Reference Ranges for Spirometry Across All Ages. Am J Respir Crit Care Med 2008:177:253-60.

37 Zhao CN, Fan Y, Huang JJ, et al. The Association of GSDMB and ORMDL3 Gene Polymorphisms With Asthma: A Meta-Analysis. Allergy Asthma Immunol Res 2015;7:175-85.

38 Yu J, Kang MJ, Kim BJ, et al. Polymorphisms in GSDMA and GSDMB are associated with asthma susceptibility, atopy and BHR. Pediatr Pulmonol 2011;46:701-8.

39 Sy HY, Ko FW, Chu HY, et al. Asthma and bronchodilator responsiveness are associated with polymorphic markers of ARG1, CRHR2 and chromosome 17q21. Pharmacogenet Genomics 2012;22:517-24.

40 Loss GJ, Depner M, Hose AJ, et al. The Early Development of Wheeze. Environmental Determinants and Genetic Susceptibility at 17q21. Am J Respir Crit Care Med 2016;193:889-97.

41 Frey U, Suki B. Complexity of chronic asthma and chronic obstructive pulmonary disease: implications for risk assessment, and disease progression and control. Lancet 2008;372:1088-99.

42 Frey U, Latzin $\mathrm{P}$, Usemann J, et al. Asthma and obesity in children: current evidence and potential systems biology approaches. Allergy 2015;70:26-40. 